\title{
Adaptive Backstepping Control of Electrical Transmission Drives with Elastic, Unknown Backlash and Coulomb Friction Nonlinearity
}

\author{
Huỳnh Văn Đông*, Trần Xuân Kiên**, Nguyễn Công Định***
}

Abstract: In this paper, we present a new scheme to design an adaptive controller for uncertain nonlinear systems with unknown backlash, Coulomb friction nonlinearity. The control design is achieved by introducing a smooth approximate backlash model and certain well defined functions and by using backstepping technique. It is shown that the proposed controller can guarantee that the system is global asymptotic stable.

Keywords: Adaptive control, backstepping, backlash, Coulomb friction, dead-zone, nonlinear systems, stability.

\section{Introduction}

Electrical transmission drives is an important part of a control system, which pass the control command from the controller to the objects. Conventionally, for convenience in designing the controller, the effects of nonlinear backlash, deadzone and friction are usually ignored. However, very often, the mentioned above parameters exist in many devices such as gearbox, transmission shaft, valve (hydraulic), DC servo motor, and so on. They are nonlinear elements, and can change from time to time, causing different limitations of quality of the whole system.

Research on Electrical Transmission Drives, which includes nonlinear backlash, dead-zone and friction, is a hot topic. The target is to improve the quality of the system based on looking at the useful nonlinear characteristic of the system. Current researches on two-mass systems can be referred to in [2]-[18].

The researches and estimations about the systems, where exist backlash and friction, can be seen in [10], [11], [12], [13]. The controller based on sliding mode for two-mass systems is introduced in [2], robust control is used in [3], [5]. Other methods based on PI control are shown in [17], PD/PI associated with Fuzzy is in[18], Fuzzy based on Takagi-Sugeno model is in [7], [17], Kalman filter is shown in [15], accurate linearization is in [4], reference model building with parameter adjustment is in [13], linearization is in [14], and backstepping is introduced in [9].

In [9] and [18], model of the plant is built, taking into consideration the parameter resilience, ignoring dead-zone and friction moment. In [14], the nonlinear elements, such as dead-zone and friction, are linearized by secants method.

This paper shows the study of common nonlinear class, as in [8]. Backlash and friction are in two differential equations of the system. The existence of backlash and friction causes difficulties for the development of the controller. A new model which smoothes backlash is chosen, and the controller is built based on 
recursive backstepping design. Nonlinear parameters are smoothed, continued and can be differentiated. In this paper, instead of concerning the effects of nonlinear backlash, resilience and friction as limited noises (as in [10], [11], [12], [13]), they are included in controller design.

Research on system, which includes nonlinear parameters, improves the quality and stability of the system. The backstepping controller, which is designed with two adapt laws for unknown parameters, is shown and it guarantees that the system is global asymptotic stable.

\section{Model of Nonlinear Electrical Transmission Drives}

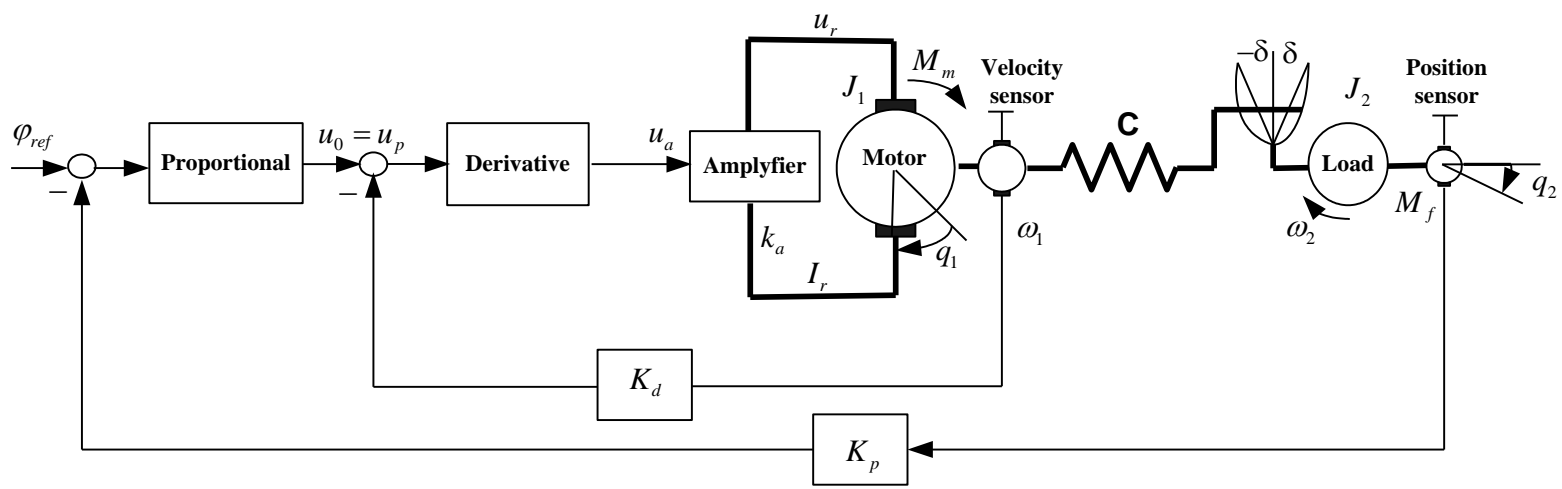

Fig.1. A schematic diagram of the nonlinear electrical transmission drives with PID controller

$$
\left\{\begin{array}{l}
\dot{\omega}_{2}=J_{2}^{-1}\left(T_{s}-M_{f}\right) ; \dot{M}_{y}=C\left(\omega_{1}-\omega_{2}\right) ; \\
\dot{\omega}_{1}=J_{1}^{-1}\left(M_{m}-T_{s}\right) ; \\
M_{m}=k_{m} I_{r} \quad ; \quad u_{r}=k_{a} u_{a} ; \quad I_{r}=\frac{k_{a} u_{a}}{R}-\frac{k_{e} \omega_{1}}{R} \\
\dot{q}_{1}=\omega_{1} ; \quad \dot{q}_{2}=\omega_{2}
\end{array}\right.
$$

In equation (1), $M_{y}=C\left(q_{1}-q_{2}\right)$ is elastic moment $[\mathrm{Nm}]$, when elastic connection is without backlash; $T_{s}$ is elastic moment $[\mathrm{Nm}]$, with backlash $2 \delta \quad[\mathrm{rad}]$ in elastic connection and is nonlinear function (undifferentiable), which have the following form:

$$
T_{s}=\left\{\begin{array}{l}
M_{y}-C \delta, \quad \text { if } \quad \mathrm{M}_{\mathrm{y}} \geq C \delta \\
0, \quad \text { if }\left|M_{y}\right|<C \delta \\
M_{y}+C \delta, \quad \text { if } \quad M_{y}<-C \delta
\end{array}\right.
$$

Where, $q_{1}, \varphi=q_{2}(\mathrm{rad})$ are angular of shaft motor and load; $\omega_{1}=\dot{q}_{1}, \omega_{2}=\dot{q}_{2}[\mathrm{rad} / \mathrm{s}]$ are the motor and load angular speeds; $J_{1}, J_{2}\left[\mathrm{kgm}^{2}\right]$ are the motor and load moments of inertia; $C[\mathrm{Nm} / \mathrm{rad}]$ is the spring constant; $M_{m}[\mathrm{Nm}]$ is the motor torque, $k_{e}[\mathrm{Vs} / \mathrm{rad}]$ is the motor's torque constant; $k_{m}$ is constant; $R[\Omega]$ is 
the armature coil resistance; $K_{p}, \quad K_{d}$ are proportional and derivative gains; $u_{p}[V]$ is output voltage of proportional controller; $u_{a}[V]$ is output voltage of derivative controller; $u_{r}[V]$ is the motor armature voltage; $I_{r}[A]$ is the armature current; $\varphi_{r e f}[\mathrm{rad}]$ is reference angular; $u_{0}=u_{p}[\mathrm{~V}]$ is signal control which follows reference program (for speed loop, it is output signal of positional controller); $M_{f}[\mathrm{Nm}]-$ Coulomb friction, from [8], we obtain:

$M_{f}=\alpha \operatorname{sign}\left(\omega_{2}\right)$

$\alpha$ - positive constant; $\operatorname{sign}()-$. sign function of (.).

We can rewrite (2) in form as:

$$
T_{s}=\left\{\begin{array}{l}
C\left[\left(q_{1}-q_{2}\right)-\delta\right], \quad \text { if } \quad C\left(q_{1}-q_{2}\right) \geq C \delta \\
0, \quad \text { if } \quad\left|C\left(q_{1}-q_{2}\right)\right|<C \delta \\
C\left[\left(q_{1}-q_{2}\right)+\delta\right], \quad \text { if } \quad C\left(q_{1}-q_{2}\right)<-C \delta
\end{array}\right.
$$

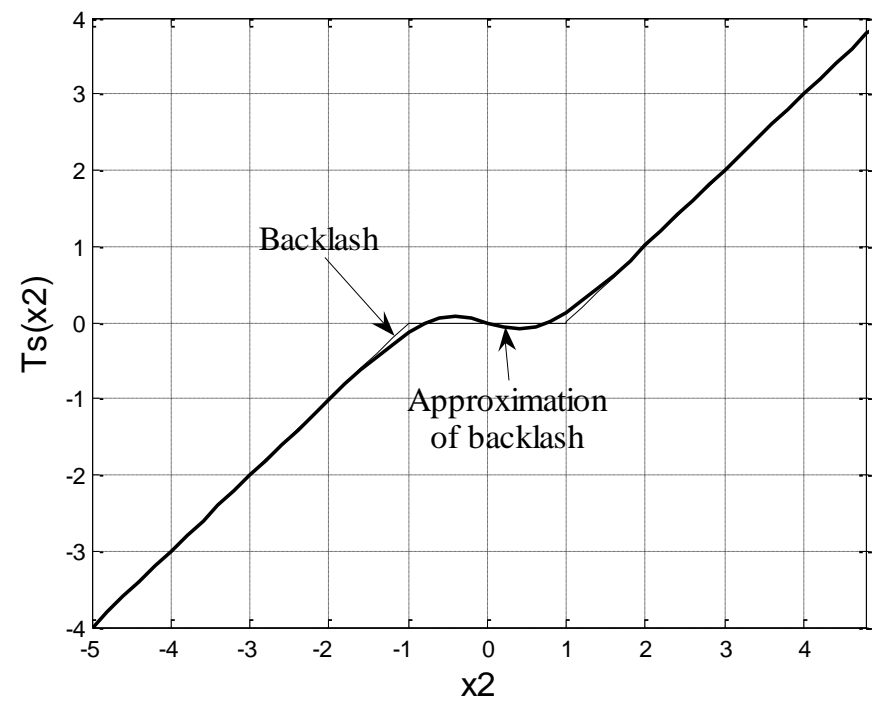

Fig 2a. Model of backlash and smooth approximation

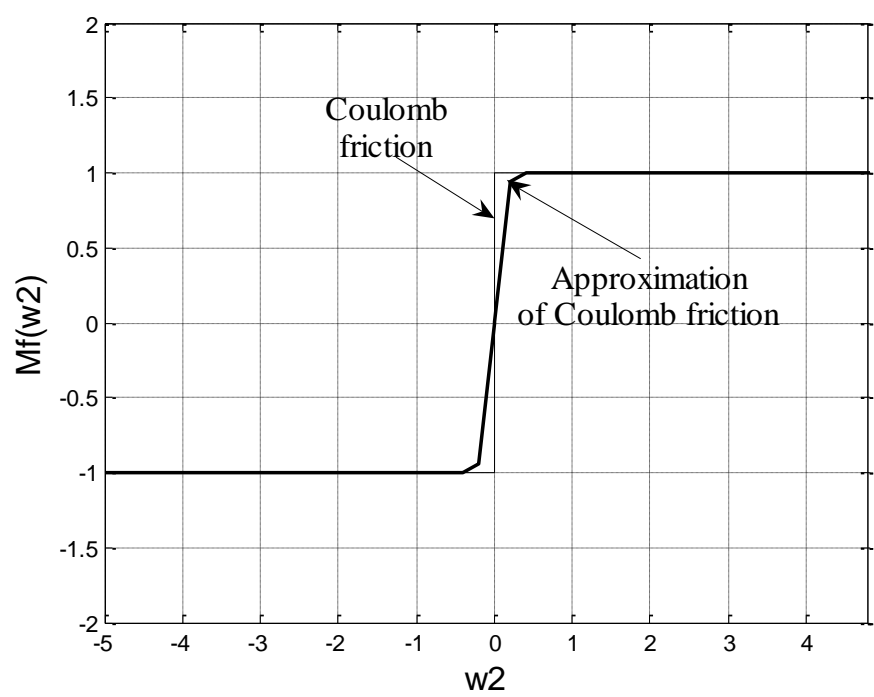

Fig 2b. Model of Coulomb friction and smooth approximation 
Set:

$x_{2}=q_{1}-q_{2}$

We obtain:

$T_{s}=\left\{\begin{array}{l}C\left(x_{2}-\delta\right), \quad \text { if } \quad x_{2} \geq \delta \\ 0, \quad \text { if } \quad\left|x_{2}\right|<\delta \\ C\left(x_{2}+\delta\right), \quad \text { if } \quad x_{2}<-\delta\end{array}\right.$

In [3] and [4], we can approximate (6) by smooth function as:

$T_{s}=C\left[x_{2}-\delta \tanh \left(a x_{2}\right)\right]$

In [6] and [8], we can approximate (3) as:

$M_{m s}=\alpha \operatorname{sign}\left(\omega_{2}\right)=\alpha \tanh \left(b \omega_{2}\right)$

In (7) and (8), $a, b$ are positive numbers, which can be chosen when designing (in figure $2 \mathrm{a}$, choose $a=1,25$; in figure $2 \mathrm{~b}$, choose $b=9$ ).

Set $x_{1}=\omega_{2} ; \quad x_{2}=q_{1}-q_{2} ; \quad x_{3}=\omega_{1}-\omega_{2}$, we can rewrite (1) as:

$\dot{x}_{1}=\frac{C}{J_{2}}\left[x_{2}-\delta \tanh \left(a x_{2}\right)\right]-\frac{\alpha}{J_{2}} \tanh \left(b x_{1}\right)$

$\dot{x}_{2}=x_{3}$

$\dot{x}_{3}=-\left(\frac{C}{J_{1}}+\frac{C}{J_{2}}\right)\left[x_{2}-\delta \tanh \left(a x_{2}\right)\right]+\frac{\alpha}{J_{2}} \tanh \left(b x_{1}\right)-\frac{k_{e} k_{m}}{J_{1} R}\left(x_{1}+x_{3}\right)+\frac{k_{m} k_{a}}{J_{1} R} u_{a}$

$y=x_{1}$

Set: $a_{1}=\frac{C}{J_{2}} ; \quad \theta_{2}=\frac{\alpha}{J_{2}} ; \quad a_{2}=-\left(\frac{C}{J_{1}}+\frac{C}{J_{2}}\right) ; \quad a_{3}=\frac{k_{e} k_{m}}{J_{1} R} ; \quad a_{4}=\frac{k_{m} k_{a}}{J_{1} R} ; \quad \delta=\theta_{1}$,

we obtain:

$a_{1} ; a_{2} ; a_{3} ; a_{4}$ are known parameters (can be measured); unknown parameters are: $\theta_{1}$ - width of backlash, $\theta_{2}$ - including Coulomb friction.

We can rewrite (9) as:

$\dot{x}_{1}=a_{1}\left[x_{2}-\theta_{1} \tanh \left(a x_{2}\right)\right]-\theta_{2} \tanh \left(b x_{1}\right)$

$\dot{x}_{2}=x_{3}$

$\dot{x}_{3}=a_{2}\left[x_{2}-\theta_{1} \tanh \left(a x_{2}\right)\right]+\theta_{2} \tanh \left(b x_{1}\right)-a_{3}\left(x_{1}+x_{3}\right)+a_{4} u_{a}$

$y=x_{1}$

For system described by (10), we can design adaptive backstepping controller for system (1) based on theory introduced in [1].

\section{Design of Adaptive Backstepping Controller:}

\section{Step 1:}

Set the system's final output $y=x_{1}=\omega_{2}$, because this speed can not be measured directly when variation of elastic is included, name its asymptotic value is $y_{d}$, adjusting error $z_{1}$ can be calculated as:

$$
z_{1}=y-y_{d}=x_{1}-y_{d}
$$

Assume that $\dot{y}_{d}=0$, we obtain: $\dot{z}_{1}=\dot{x}_{1}=a_{1}\left[x_{2}-\theta_{1} \tanh \left(a x_{2}\right)\right]-\theta_{2} \tanh \left(b x_{1}\right)$. 
Because $\theta_{1}, \theta_{2}$ are unknown parameters, we denote their corresponding estimated parameters are $\hat{\theta}_{1}, \hat{\theta}_{2}$, tracking errors are:

$$
\begin{aligned}
& \xi_{1}=\theta_{1}-\hat{\theta}_{1} \text { or } \theta_{1}=\hat{\theta}_{1}+\xi_{1} \\
& \xi_{2}=\theta_{2}-\hat{\theta}_{2} \text { or } \theta_{2}=\hat{\theta}_{2}+\xi_{2}
\end{aligned}
$$

We choose Lyapunov function for $z_{1}$ is: $V_{1}=\frac{1}{2 a_{1}} z_{1}^{2}+\frac{1}{2 \gamma} \xi_{1}^{2}+\frac{1}{2 \beta} \xi_{2}^{2}$.

Where, $\gamma, \beta$ are adaptation gains.

Differentiating of $V_{1}$ as:

$$
\begin{aligned}
& \dot{V}_{1}=\frac{1}{a_{1}} z_{1} \dot{z}_{1}+\frac{1}{\gamma} \xi_{1} \dot{\xi}_{1}+\frac{1}{\beta} \xi_{2} \dot{\xi}_{2}= \\
& =\frac{1}{a_{1}} z_{1}\left\{a_{1}\left[x_{2}-\theta_{1} \tanh \left(a x_{2}\right)\right]-\theta_{2} \tanh \left(b x_{1}\right)\right\}+\frac{1}{\gamma} \xi_{1} \dot{\xi}_{1}+\frac{1}{\beta} \xi_{2} \dot{\xi}_{2} \\
& =z_{1}\left\{\left[x_{2}-\left(\xi_{1}+\hat{\theta}_{1}\right) \tanh \left(a x_{2}\right)\right]-\frac{1}{a_{1}}\left(\xi_{2}+\hat{\theta}_{2}\right) \tanh \left(b x_{1}\right)\right\}+\frac{1}{\gamma} \xi_{1}\left(-\dot{\hat{\theta}}_{1}\right)+\frac{1}{\beta} \xi_{2}\left(-\dot{\hat{\theta}}_{2}\right) \\
& =z_{1}\left\{\left[x_{2}-\hat{\theta}_{1} \tanh \left(a x_{2}\right)\right]-\frac{1}{a_{1}} \hat{\theta}_{2} \tanh \left(b x_{1}\right)\right\}-z_{1} \xi_{1} \tanh \left(a x_{2}\right)+\frac{1}{\gamma} \xi_{1}\left(-\dot{\hat{\theta}}_{1}\right)-\frac{z_{1}}{a_{1}} \xi_{2} \tanh \left(b x_{1}\right)+\frac{1}{\beta} \xi_{2}\left(-\dot{\hat{\theta}}_{2}\right) \\
& =z_{1}\left\{\left(x_{2}-\alpha_{1}\right)+\alpha_{1}-\hat{\theta}_{1} \tanh \left(a x_{2}\right)-\frac{1}{a_{1}} \hat{\theta}_{2} \tanh \left(b x_{1}\right)\right\}-\xi_{1}\left[z_{1} \tanh \left(a x_{2}\right)+\frac{1}{\gamma} \dot{\hat{\theta}}_{1}\right]-\xi_{2}\left[\frac{z_{1}}{a_{1}} \tanh \left(b x_{1}\right)+\frac{1}{\beta} \dot{\hat{\theta}}_{2}\right]
\end{aligned}
$$

We choose the first virtual control $\alpha_{1}$ is:

$$
\begin{aligned}
& \alpha_{1}=-c_{1} z_{1}+\hat{\theta}_{1} \tanh \left(a x_{2}\right)+\frac{1}{a_{1}} \hat{\theta}_{2} \tanh \left(b x_{1}\right) \\
& \dot{V}_{1}=-c_{1} z_{1}^{2}+z_{1} z_{2}-\xi_{1}\left[z_{1} \tanh \left(a x_{2}\right)+\frac{1}{\gamma} \dot{\hat{\theta}}_{1}\right]-\xi_{2}\left[\frac{z_{1}}{a_{1}} \tanh \left(b x_{1}\right)+\frac{1}{\beta} \dot{\hat{\theta}}_{2}\right]
\end{aligned}
$$

\section{Step 2:}

$$
\begin{aligned}
& V_{2}=V_{1}+\frac{1}{2} z_{2}^{2} \quad \text { or } \\
& \dot{V}_{2}=\dot{V}_{1}+z_{2} \dot{z}_{2}=-c_{1} z_{1}^{2}+z_{2}\left(z_{1}+\dot{z}_{2}\right)-\xi_{1}\left[z_{1} \tanh \left(a x_{2}\right)+\frac{1}{\gamma} \dot{\hat{\theta}}_{1}\right]-\xi_{2}\left[\frac{z_{1}}{a_{1}} \tanh \left(b x_{1}\right)+\frac{1}{\beta} \dot{\hat{\theta}}_{2}\right]
\end{aligned}
$$

Expanding the $\left(z_{1}+\dot{z}_{2}\right)$ term:

$$
z_{1}+\dot{z}_{2}=z_{1}+\dot{x}_{2}-\ddot{y}_{d}-\dot{\alpha}_{1}\left(x_{1}, y_{d}, \hat{\theta}_{1}, \hat{\theta}_{2}, x_{2}\right)=z_{1}+x_{3}-\dot{\alpha}_{1}\left(x_{1}, y_{d}, \hat{\theta}_{1}, \hat{\theta}_{2}, x_{2}\right)
$$

From (14), we can write:

$\alpha_{1}=-c_{1} z_{1}+\hat{\theta}_{1} \tanh \left(a x_{2}\right)+\frac{1}{a_{1}} \hat{\theta}_{2} \tanh \left(b x_{1}\right)=-c_{1} x_{1}+c_{1} y_{d}+\hat{\theta}_{1} \tanh \left(a x_{2}\right)+\frac{1}{a_{1}} \hat{\theta}_{2} \tanh \left(b x_{1}\right)$

$\frac{\partial \alpha_{1}}{\partial x_{1}}=-c_{1}+\frac{b}{a_{1}} \hat{\theta}_{2}\left[1-\tanh ^{2}\left(b x_{1}\right)\right]$

$\frac{\partial \alpha_{1}}{\partial y_{d}}=0$

$(20) ; \quad \frac{\partial \alpha_{1}}{\partial \hat{\theta}_{1}}=\tanh \left(a x_{2}\right)$ 


$$
\frac{\partial \alpha_{1}}{\partial \hat{\theta}_{2}}=\frac{1}{a_{1}} \tanh \left(b x_{1}\right) \quad(22) ; \quad \frac{\partial \alpha_{1}}{\partial x_{2}}=a \hat{\theta}\left[1-\tanh ^{2}\left(a x_{2}\right)\right]
$$

Substituting (19)-(23) into equation (17), we obtain:

$$
\begin{aligned}
z_{1}+\dot{z}_{2} & =z_{1}+\left(x_{3}-\alpha_{2}\right)+\alpha_{2}+c_{1}-\frac{b}{a_{1}} \hat{\theta}_{2}\left[1-\tanh ^{2}\left(b x_{1}\right)\right]-\tanh \left(a x_{2}\right)-\frac{1}{a_{1}} \tanh \left(b x_{1}\right)-a \hat{\theta}_{1}\left[1-\tanh ^{2}\left(a x_{2}\right)\right] \\
& =z_{1}+z_{3}+\alpha_{2}+c_{1}-\frac{b}{a_{1}} \hat{\theta}_{2}\left[1-\tanh ^{2}\left(b x_{1}\right)\right]-\tanh \left(a x_{2}\right)-\frac{1}{a_{1}} \tanh \left(b x_{1}\right)-a \hat{\theta}_{1}\left[1-\tanh ^{2}\left(a x_{2}\right)\right]
\end{aligned}
$$

We choose:

$$
\begin{aligned}
& \alpha_{2}=-c_{2} z_{2}-\left\{z_{1}+c_{1}-\frac{b}{a_{1}} \hat{\theta}_{2}\left[1-\tanh ^{2}\left(b x_{1}\right)\right]-\tanh \left(a x_{2}\right)-\frac{1}{a_{1}} \tanh \left(b x_{1}\right)-a \hat{\theta}_{1}\left[1-\tanh ^{2}\left(a x_{2}\right)\right]\right\} \\
& \dot{V}_{2}=\dot{V}_{1}+z_{2} \dot{z}_{2}=-c_{1} z_{1}^{2}-c_{2} z_{2}^{2}+z_{2} z_{3}-\xi_{1}\left[z_{1} \tanh \left(a x_{2}\right)+\frac{1}{\gamma} \dot{\hat{\theta}}_{1}\right]-\xi_{2}\left[\frac{z_{1}}{a_{1}} \tanh \left(b x_{1}\right)+\frac{1}{\beta} \dot{\hat{\theta}}_{2}\right]
\end{aligned}
$$

\section{Step 3:}

$$
\begin{aligned}
V_{3} & =V_{2}+\frac{1}{2} z_{3}^{2} \quad \text { or } \\
\dot{V}_{3} & =\dot{V}_{2}+z_{3} \dot{z}_{3} \\
& =-c_{1} z_{1}^{2}-c_{2} z_{2}^{2}+z_{3}\left(z_{2}+\dot{z}_{3}\right)-\xi_{1}\left[z_{1} \tanh \left(a x_{2}\right)+\frac{1}{\gamma} \dot{\hat{\theta}}_{1}\right]-\xi_{2}\left[\frac{z_{1}}{a_{1}} \tanh \left(b x_{1}\right)+\frac{1}{\beta} \dot{\hat{\theta}}_{2}\right]
\end{aligned}
$$

Again expanding the $\left(z_{2}+\dot{z}_{3}\right)$ term:

$$
\begin{aligned}
& z_{2}+\dot{z}_{3}=z_{2}+\dot{x}_{3}-\dot{\alpha}_{2}\left(x_{1}, y_{d}, \hat{\theta}_{1}, \hat{\theta}_{2}, x_{2}\right) \\
& =z_{2}+a_{2}\left[x_{2}-\theta_{1} \tanh \left(a x_{2}\right)\right]+\theta_{2} \tanh \left(b x_{1}\right)-a_{3}\left(x_{1}+x_{3}\right)+a_{4} u_{a}-\dot{\alpha}_{2}\left(x_{1}, y_{d}, \hat{\theta}_{1}, \hat{\theta}_{2}, x_{2}\right) \\
& \alpha_{2}=-c_{2}\left(x_{2}-\dot{y}_{d}-\alpha_{1}\right) \\
& -\left\{z_{1}+c_{1}-\frac{b}{a_{1}} \hat{\theta}_{2}\left[1-\tanh ^{2}\left(b x_{1}\right)\right]-\tanh \left(a x_{2}\right)-\frac{1}{a_{1}} \tanh \left(b x_{1}\right)-a \hat{\theta}_{1}\left[1-\tanh ^{2}\left(a x_{2}\right)\right]\right\} \\
& \alpha_{2}=-c_{2} x_{2}+c_{1} c_{2} z_{1}-c_{2} \hat{\theta}_{1} \tanh \left(a x_{2}\right)-c_{2} \frac{1}{a_{1}} \hat{\theta}_{2} \tanh \left(b x_{1}\right) \\
& -\left\{z_{1}+c_{1}-\frac{b}{a_{1}} \hat{\theta}_{2}\left[1-\tanh ^{2}\left(b x_{1}\right)\right]-\tanh \left(a x_{2}\right)-\frac{1}{a_{1}} \tanh \left(b x_{1}\right)-a \hat{\theta}_{1}\left[1-\tanh ^{2}\left(a x_{2}\right)\right]\right\} \\
& \alpha_{2}=-c_{2} x_{2}+c_{1} c_{2}\left(x_{1}-y_{d}\right)-c_{2} \hat{\theta}_{1} \tanh \left(a x_{2}\right)-c_{2} \frac{1}{a_{1}} \hat{\theta}_{2} \tanh \left(b x_{1}\right) \\
& -\left\{\left(x_{1}-y_{d}\right)+c_{1}-\frac{b}{a_{1}} \hat{\theta}_{2}\left[1-\tanh ^{2}\left(b x_{1}\right)\right]-\tanh \left(a x_{2}\right)-\frac{1}{a_{1}} \tanh \left(b x_{1}\right)-a \hat{\theta}_{1}\left[1-\tanh ^{2}\left(a x_{2}\right)\right]\right\}
\end{aligned}
$$

We calculate the partial derivatives of $\alpha_{2}$ :

$$
\begin{aligned}
\frac{\partial \alpha_{2}}{\partial x_{1}}=c_{1} c_{2}-\frac{b c_{2}}{a_{1}} \hat{\theta}_{2}\left[1-\tanh ^{2}\left(b x_{1}\right)\right]-1-\frac{2 b^{2}}{a_{1}} \hat{\theta}_{2} \tanh \left(b x_{1}\right)\left[1-\tanh ^{2}\left(b x_{1}\right)\right]+\frac{b}{a_{1}}\left[1-\tanh ^{2}\left(b x_{1}\right)\right] \\
\frac{\partial \alpha_{2}}{\partial y_{d}}=0 \\
\frac{\partial \alpha_{2}}{\partial x_{2}}=-c_{2}-c_{2} a \hat{\theta}_{1}\left[1-\tanh ^{2}\left(a x_{2}\right)\right]+a\left[1-\tanh ^{2}\left(a x_{2}\right)\right]-2 a^{2} \hat{\theta}_{1} \tanh \left(a x_{2}\right)\left[1-\tanh ^{2}\left(a x_{2}\right)\right]
\end{aligned}
$$




$$
\begin{aligned}
& \frac{\partial \alpha_{2}}{\partial \hat{\theta}_{1}}=-c_{2} \tanh \left(a x_{2}\right)+a\left[1-\tanh ^{2}\left(a x_{2}\right)\right] \\
& \frac{\partial \alpha_{2}}{\partial \hat{\theta}_{2}}=-\frac{c_{2}}{a_{1}} \tanh \left(b x_{1}\right)+\frac{b}{a_{1}}\left[1-\tanh ^{2}\left(b x_{1}\right)\right]
\end{aligned}
$$

Substituting (31)-(35) into equation (27), we obtain:

$z_{2}+\dot{z}_{3}=z_{2}+a_{2}\left[x_{2}-\left(\xi_{1}+\hat{\theta}_{1}\right) \tanh \left(a x_{2}\right)\right]+\left(\xi_{2}+\hat{\theta}_{2}\right) \tanh \left(b x_{1}\right)-a_{3}\left(x_{1}+x_{3}\right)+a_{4} u_{a}-\dot{\alpha}_{2}\left(x_{1}, y_{d}, \hat{\theta}_{1}, \hat{\theta}_{2}, x_{2}\right)$

$=z_{2}+a_{2} x_{2}-a_{2} \hat{\theta}_{1} \tanh \left(a x_{2}\right)-a_{2} \xi_{1} \tanh \left(a x_{2}\right)+\hat{\theta}_{2} \tanh \left(b x_{1}\right)+\xi_{2} \tanh \left(b x_{1}\right)-a_{3}\left(x_{1}+x_{3}\right)+a_{4} u_{a}$

$-c_{1} c_{2}+\frac{b c_{2}}{a_{1}} \hat{\theta}_{2}\left[1-\tanh ^{2}\left(b x_{1}\right)\right]+1+\frac{2 b^{2}}{a_{1}} \hat{\theta}_{2} \tanh \left(b x_{1}\right)\left[1-\tanh ^{2}\left(b x_{1}\right)\right]-\frac{b}{a_{1}}\left[1-\tanh ^{2}\left(b x_{1}\right)\right]$

$+c_{2}+c_{2} a \hat{\theta}_{1}\left[1-\tanh ^{2}\left(a x_{2}\right)\right]-a\left[1-\tanh ^{2}\left(a x_{2}\right)\right]+2 a^{2} \hat{\theta}_{1} \tanh \left(a x_{2}\right)\left[1-\tanh ^{2}\left(a x_{2}\right)\right]$

$+c_{2} \tanh \left(a x_{2}\right)-a\left[1-\tanh ^{2}\left(a x_{2}\right)\right]+\frac{c_{2}}{a_{1}} \tanh \left(b x_{1}\right)-\frac{b}{a_{1}}\left[1-\tanh ^{2}\left(b x_{1}\right)\right]$

\section{Choose:}

$$
\begin{aligned}
& a_{4} u_{a}=-c_{3} z_{3}-\left\{z_{2}+a_{2} x_{2}-a_{2} \hat{\theta}_{1} \tanh \left(a x_{2}\right)+\hat{\theta}_{2} \tanh \left(b x_{1}\right)-a_{3}\left(x_{1}+x_{3}\right)\right. \\
& -c_{1} c_{2}+\frac{b c_{2}}{a_{1}} \hat{\theta}_{2}\left[1-\tanh ^{2}\left(b x_{1}\right)\right]+1+\frac{2 b^{2}}{a_{1}} \hat{\theta}_{2} \tanh \left(b x_{1}\right)\left[1-\tanh ^{2}\left(b x_{1}\right)\right]-\frac{2 b}{a_{1}}\left[1-\tanh ^{2}\left(b x_{1}\right)\right] \\
& +c_{2}+c_{2} a \hat{\theta}_{1}\left[1-\tanh ^{2}\left(a x_{2}\right)\right]-2 a\left[1-\tanh ^{2}\left(a x_{2}\right)\right]+2 a^{2} \hat{\theta}_{1} \tanh \left(a x_{2}\right)\left[1-\tanh ^{2}\left(a x_{2}\right)\right] \\
& \left.+c_{2} \tanh \left(a x_{2}\right)+\frac{c_{2}}{a_{1}} \tanh \left(b x_{1}\right)\right\} \\
& \quad u_{a}=\frac{1}{a_{4}}\left\{-c_{3} z_{3}-z_{2}-a_{2} x_{2}+a_{2} \hat{\theta}_{1} \tanh \left(a x_{2}\right)-\hat{\theta}_{2} \tanh \left(b x_{1}\right)+a_{3}\left(x_{1}+x_{3}\right)\right. \\
& +c_{1} c_{2}-\frac{b c_{2}}{a_{1}} \hat{\theta}_{2}\left[1-\tanh ^{2}\left(b x_{1}\right)\right]-1-\frac{2 b^{2}}{a_{1}} \hat{\theta}_{2} \tanh \left(b x_{1}\right)\left[1-\tanh ^{2}\left(b x_{1}\right)\right]+\frac{2 b}{a_{1}}\left[1-\tanh ^{2}\left(b x_{1}\right)\right] \\
& -c_{2}-c_{2} a \hat{\theta}_{1}\left[1-\tanh ^{2}\left(a x_{2}\right)\right]+2 a\left[1-\tanh { }^{2}\left(a x_{2}\right)\right]-2 a^{2} \hat{\theta}_{1} \tanh ^{2}\left(a x_{2}\right)\left[1-\tanh ^{2}\left(a x_{2}\right)\right] \\
& \left.-c_{2} \tanh \left(a x_{2}\right)-\frac{c_{2}}{a_{1}} \tanh \left(b x_{1}\right)\right\}
\end{aligned}
$$

Substituting (38) into (36), then (36) into (26), we obtain:

$\dot{V}_{3}=-c_{1} z_{1}^{2}-c_{2} z_{2}^{2}+z_{3}\left[-c_{3} z_{3}-a_{2} \xi_{1} \tanh \left(a x_{2}\right)+\xi_{2} \tanh \left(b x_{1}\right)\right]$

$$
-\xi_{1}\left[z_{1} \tanh \left(a x_{2}\right)+\frac{1}{\gamma} \dot{\hat{\theta}}_{1}\right]-\xi_{2}\left[\frac{z_{1}}{a_{1}} \tanh \left(b x_{1}\right)+\frac{1}{\beta} \dot{\hat{\theta}}_{2}\right]
$$

$\dot{V}_{3}=-c_{1} z_{1}^{2}-c_{2} z_{2}^{2}-c_{3} z_{3}^{2}$

$-\xi_{1}\left[z_{3} a_{2} \tanh \left(a x_{2}\right)+z_{1} \tanh \left(a x_{2}\right)+\frac{1}{\gamma} \dot{\hat{\theta}}_{1}\right]-\xi_{2}\left[-z_{3} \tanh \left(b x_{1}\right)+\frac{z_{1}}{a_{1}} \tanh \left(b x_{1}\right)+\frac{1}{\beta} \dot{\hat{\theta}}_{2}\right]$

$\dot{\hat{\theta}}_{1}=-\gamma z_{3} a_{2} \tanh \left(a x_{2}\right)-\gamma z_{1} \tanh \left(a x_{2}\right)$

$\dot{\hat{\theta}}_{2}=z_{3} \beta \tanh \left(b x_{1}\right)-\frac{z_{1} \beta}{a_{1}} \tanh \left(b x_{1}\right)$

$\dot{V}_{3}=-c_{1} z_{1}^{2}-c_{2} z_{2}^{2}-c_{3} z_{3}^{2}<0$ with $c_{1}>0, \quad c_{2}>0, \quad c_{3}>0$. 
To conclude, when $c_{1}>0, c_{2}>0, c_{3}>0$, with control law (38) and adaptive law (41), system (1) becomes GAS.

\section{Simulation:}

a) Simulation in Matlab-Simulink:

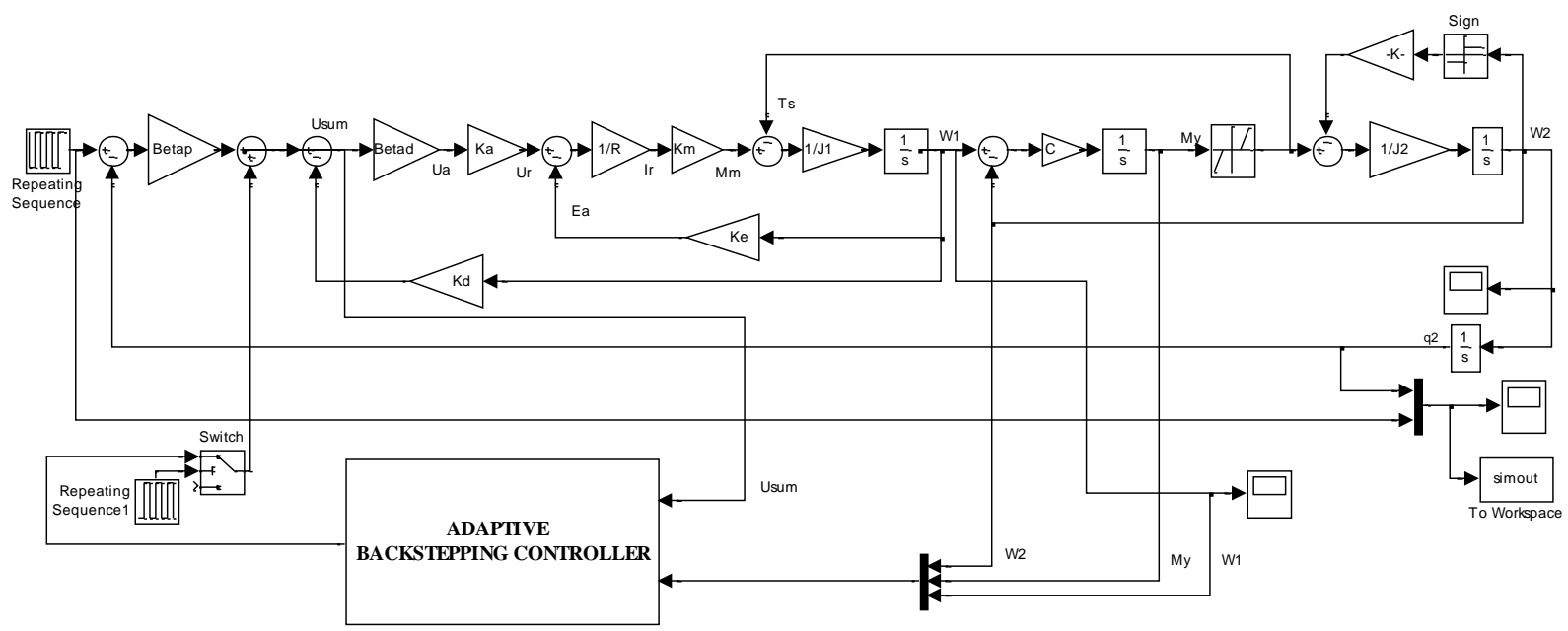

Fig 3a. Simulating in Matlab-Simulink backstepping control (38) and adaptive law (41) for nonlinear electrical transmission drives (1)

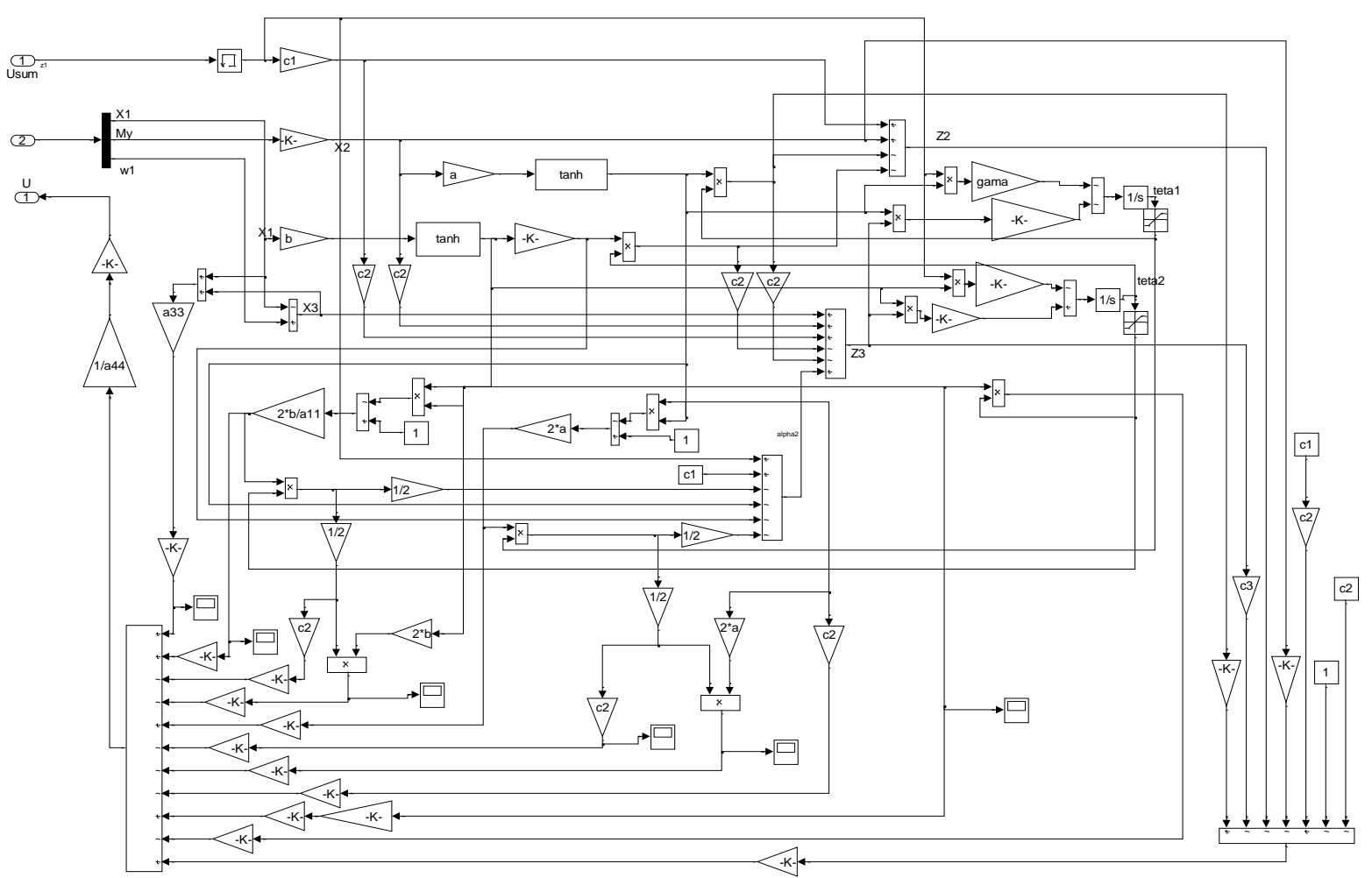

Fig 3b. Adaptive backstepping controller (38) 


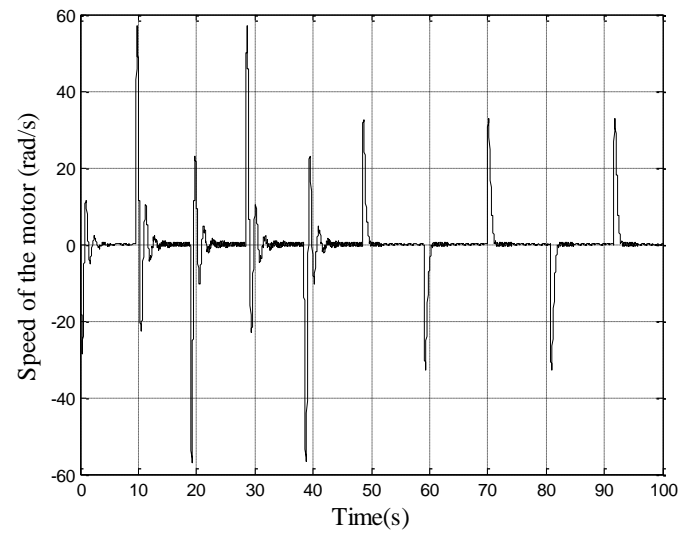

Fig 3c. Speed of motor

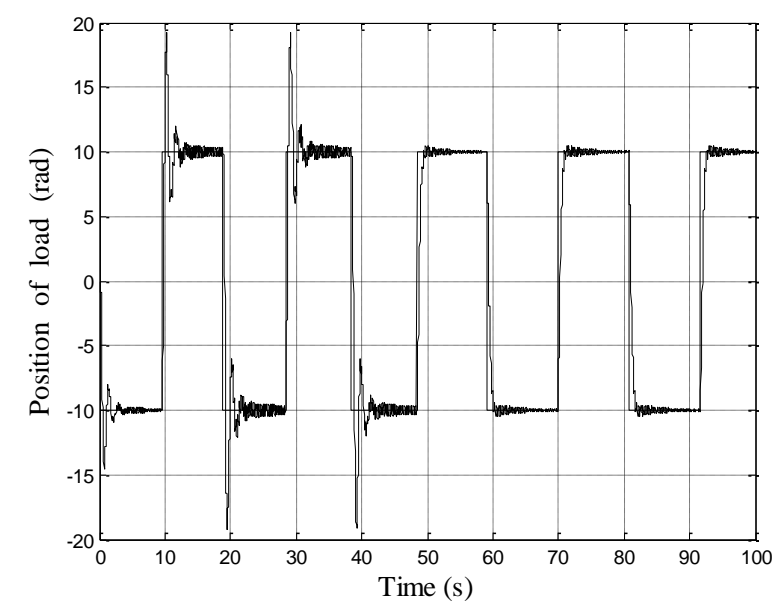

Fig 3d. Position of load

\section{b) Simulation on real model in lab:}

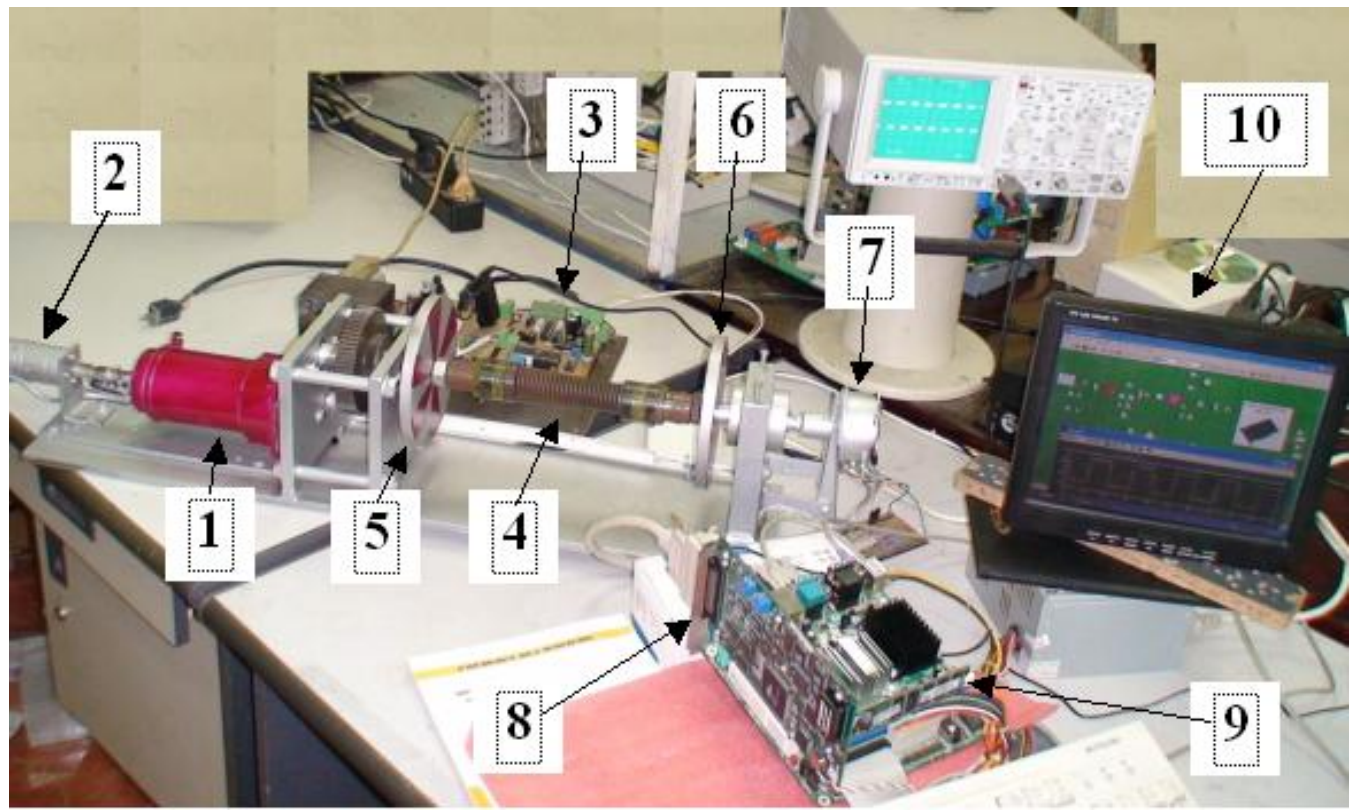

Fig 4a. Experimental model of nonlinear electrical transmission drives

1- DC motor, 2- Velocity sensor $\omega_{1}, 3$ - Pulse width modulation (PWM) and power amplifier, 4- Torsion spring connecting between two masses, 5- The first mass, 6- The second mass, 7- Position sensor,

8- Card PCI 1711 Advantech, 9- Embedded computer, 10- Controling software in Matlab-Simulink.

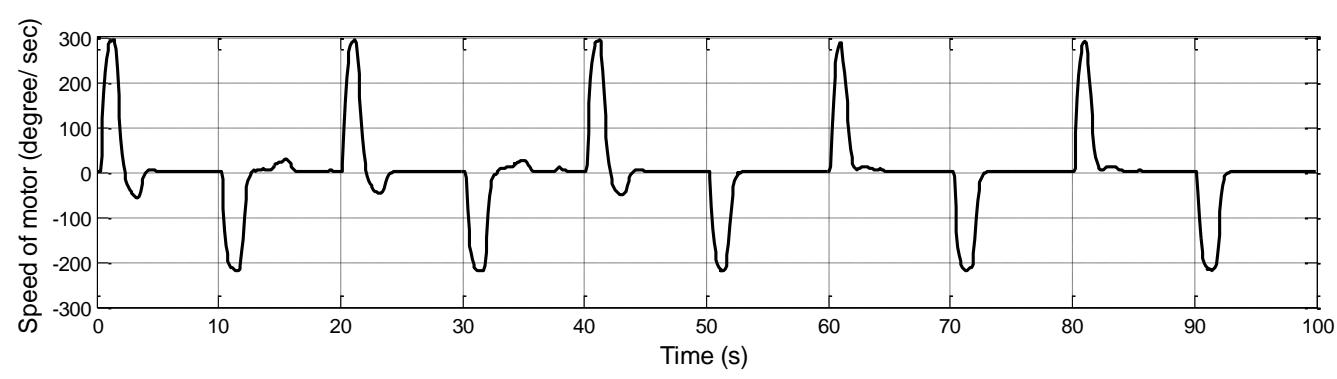

Fig 4b. Speed of motor in model $4 \mathrm{a}$ 


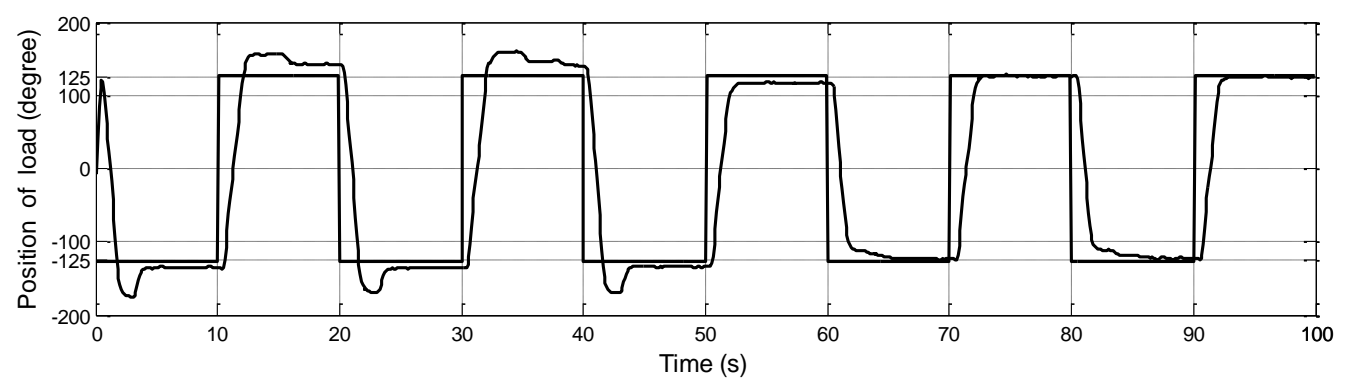

Fig 4c. Position of load in model 4a

Looking on the figures $3 \mathrm{c}, 3 \mathrm{~d}, 4 \mathrm{~b}, 4 \mathrm{c}$, during the first 50 seconds, the velocity signal is driven by the PID control, this value is fluctuated. During the next 50s, the speed is driven by the adaptive backstepping control, the speed signal is steady and the speed of motor and load follows the reference command accurately.

The comparison of the simulating results in Matlab-Simulink and on real model can conclude about the truth of the designed control algorithm.

\section{Conclusion:}

In fact, backlash, elastic and friction always exist in electro-mechanic systems. Backlash and Coulomb friction are typical nonlinear elements. They cause bad effects on system's operation quality. This can not be overcome by using the traditional controllers. By using adaptive backstepping technique, the bad effects from backlash, elastic and friction are solved. The controller has designed for the electro-mechanic object class, which includes two nonlinear masses. The controller drives the system in a "calmer" operation, also gains "good" nonlinear characteristics. Especially, it always keeps the system in global asymptote stability. 


\section{References}

[1] Miroslav Krstic', Loannis Kanellakopoulos, Petar Kokotovic'(1995), Nonlinear And Adaptive Control Design, A Wiley - Interscience Publication John Wiley \& Sons, Inc. PP 99-103.

[2] Angela Porumb (1997) "Position Control of an Elastic Two-Mass Driving System with Backlash and Friction Using a Sliding Mode Controller", Mechanics, Automatic and Robotics. Vol.2, No 7, PP 285-290.

[3] I.Schöling, B.Orlik (1999) "Robust Control of a Nonlinear Two-Mass System", University of Bremen, Germany.

[4] I.Schöling, B.Orlik (2000), "Control of a Nonlinear Two-Mass System with Uncertain Parameters and Unknown States", University of Bremen, Germany.

[5] Wolfgang Reinelt (2000), "Robust Control of a Two - Mass - Spring System Subject to Its Input Constraints", Department of Electrical Engineering/ Linköping University, Sweden.

[6] H.G Kwatny, C.Teolis, M.Macttice (2002), "Variable Structure Control of Systems with Uncertain Nonlinear Friction", Automatica 38, PP 1251-1256.

[7] Danijiel Pavkovic', Ivan Petrovic' and Nedjeljko Perie' (2002) "Fuzzy Model Predictive Control of Electrical Drives with Transmission Elasticity and Backlash". Automatica, Vol 43 No 1-2, PP 5-11.

[8] Tolgay Kara, Ilyas Eker (2003), "Nonlinear Modeling and Identification of a DC motor for Bidirectional Operation with Real Time Experiments", Energy Conversion and Management.

[9] Kang-Zhi Liu, Susumu Namiki, Hidekazu Ishii (2003), "Velocity Control of 2-MassSpring Systems with Large Load Uncertainty - An Adaptive Backstepping Control Approach".Proccedings of the 42nd IEEE, USA.

[10] Adam Lagerberg (2004), Control and Estimation of Automotive Powertrains with Backlash, Chalmers University of Technology, Sweden.

[11] Mats Tallfors (2005), Parameter Estimation and Model Based Control Design of Drive Train Systems, Sweden.

[12] R.M.R. Bruns, J.F.P.B. Diepstraten, X.G.P. Schuurbiers, J.A.G. Wouters (2006), Motion Control of Systems with Backlash, Master team project.

[13] Козлов Юрий Константинович (2005), Адаптивные электромеханическимые системы стабилизизованого наведения подвижных обьектов с упругими деформащиями. ЛЭТИ САНКТ-ПЕТЕРБУРГ.

[14] V.Kudin, J.Kolacny, A.Toropov, Ivo Pazdera (2006), "Synthesis of Lathe Cutting Force Stabilization Loop Nonlinear Suboptimal Regulator Taking into Account Nonlinear Elastic Connection". National Technical University of Ukraine, FEEA.

[15] K.Szavat, T.Orlowska-Kowalska, K.P. Dyrcz (2006), "Extended Kalman Filters in the Control Structure of Two-Mass Drive System", Wroclaw University of Technology, Poland.

[16] Achim Ilchmann, Hans Schuster (2007), "PI - Funnel Control for Two Mass System", IEEE Trans. on Automatic Control.

[17] Путов В. В, Шелудько В.Н, Лебедев В.В, Зунг Ч.А, Казаков В.П, Путов А. В, Друян Е.В (2007), “Семейство аналичитеских и интеллектуальных адаптивных систем управлениея нелинейными упругими электромеханическими обьектами". Мехатроника автоматизация управнение, N10,C.16-24.

[18] Neng-Sheng Pai, Yi-Pin Kuo (2008), "Speed Control for a Two-Mass Drive System Using Integrated Fuzzy Estimator and Hybrid Fuzzy PD/PI Controller". Journal of Physics: Conference Series 96. 
* Department of Science, Technology and Environment, Vietnam email: hdongkh@yahoo.com

** Central of Science and Technology, Vietnam

email: txkien2003@yahoo.com

*** Le Quy Don Technical University, Vietnam

email: ncdinh63@yahoo.com 